\title{
Evaluation of a Recombinant Vaccine Candidate r56Lc-1 in a Chigger Challenge Mouse Model
}

\author{
Wei-Mei Ching ${ }^{1,3^{*}}$, Woradee Lurchachaiwong ${ }^{2}$, Zhiwen Zhang ${ }^{1,3}$ Temitayo Awoyomi $^{1,3}$, Chien-Chung Chao ${ }^{1,3}$ and Anthony Schuster $^{2}$ \\ ${ }^{1}$ Viral and Rickettsial Diseases Department, Infectious Diseases Directorate, Naval Medical Research Center, Silver Spring, USA \\ ${ }^{2}$ Entomology Department, Armed Forces Research Institute of Medical Sciences, Bangkok, Thailand \\ ${ }^{3}$ Uniformed Services University of the Health Sciences, Bethesda, USA
}

"Corresponding author: Wei-Mei Ching, PhD, Viral and Rickettsial Diseases Department, Infectious Diseases Directorate, Naval Medical Research Center, 503 Robert Grant Ave, RM3N71, Silver Spring, MD 20910, USA, Tel: 301319 7438; Fax: 301319 7451; E-mail: WeiMei.Ching@med.navy.mil

Received date: 15 Sep 2014; Accepted date: 24 Oct 2014; Published date: 27 Oct 2014

Copyright: @ 2014 Ching WM, et al. This is an open-access article distributed under the terms of the Creative Commons Attribution License, which permits unrestricted use, distribution, and reproduction in any medium, provided the original author and source are credited.

\begin{abstract}
Scrub typhus, an acute, febrile disease is transmitted by the bite of an Orientia infected chigger. We evaluated the protective potential of a recombinant $56 \mathrm{kDa}$ antigen in a chigger challenge mouse model which mimics the natural transmission of Orientia. Chiggers from an L. chiangraiensis line 1 (LC-1) was chosen for this challenge because the line produces chiggers with stable infectivity of $90-100 \%$ in several generations. The $56 \mathrm{kD}$ antigen gene of $O$. tsutsugamushi was cloned into an expression vector, expressed, purified, and refolded. All ICR mice were immunized 3 times at 4 weeks intervals and challenged by placing an individual chigger in the inner ear of each mouse 4 weeks after the last immunization. Mice were immunized with adjuvant Montanide+CpG or $25 \mathrm{~g}$ of r56Lc-1 emulsion with Montanide+CpG. Both groups were challenged by an individual Lc-1 chigger (un-infected or infected). There was no death in the groups of mice challenged by an uninfected chigger, with or without the vaccine candidate r56Lc-1. There was no survival in the groups of mice immunized with adjuvant only and challenged by an infected chigger. Our data demonstrated that $\mathrm{r} 56 \mathrm{LC}-1$ provided $20-30 \%$ of protection consistently upon challenge by an infected chigger which carries the same Orientia strain based on the sequence of $56 \mathrm{kDa}$ antigen. Time to mortality was delayed in mice immunized with the vaccine candidate then challenged with infected chiggers as compared to their adjuvant counterparts Since Orientia is an intracellular pathogen, the clearance of this pathogen may heavily depend on T-cell immune responses rather than B-cell immune responses and may require a vaccine candidate that mainly promotes T-cell immunity.
\end{abstract}

Keywords: ICR mice; Leptotrombidium chiangraiensis - Line 1 (Lc-1); Chigger challenge; Mites; Scrub typhus vaccine; Mouse model; Orientia tsutsugamushi

\section{Introduction}

Scrub typhus is caused by the infection of Orientia tsutsugamushi, an obligate intracellular Gram-negative bacterium [1]. The disease is characterized by fever, rash, eschar, pneumonitis, meningitis, and in some cases, disseminated intravascular coagulation that may lead to circulatory failure [2]. It accounts for up to $23 \%$ of all febrile episodes in endemic areas of the Asia-Pacific, while recent estimations in Thailand are about $20 \%$ [3]. Scrub typhus can be fatal if left untreated $[2,4]$ and reports from India have documented $12-17 \%$ fatality rates $[5,6]$. Geographic distribution of the disease occurs within an area of about 13 million square kilometers and includes Pakistan, India and Nepal in the West to Japan in the East, and from South eastern Siberia, China, and Korea in the North to Indonesia, Philippines, Northern Australia and the intervening Pacific islands in the South [7]. The disease has been re-emerging and occurs in new areas in many countries located in the Eastern Hemisphere [8-10]. Recently, two publications have documented the presence of scrub typhus in the Middle East (United Arab) and South America (Chile) [11,12]. The presence of scrub typhus outside the traditional geographic distribution suggests an increased risk for US military personnel and travellers entering into these newly emerged regions. At this present time, no vaccine is available for protection against scrub typhus
[13-16]. In addition, evidence of antibiotic resistance cases further emphasizes the need for a scrub typhus vaccine $[17,18]$. Due to the fact that $O$. tsutsugamushi exhibits considerable strain variations, previous vaccine development has been difficult.

In the past, effective vaccination in mice has been achieved with subcutaneous biovaccines in which live organisms are combined with tetracycline or with gamma-irradiated live organisms [19,20]. Also, immunization of volunteers with live vaccine in combination with chloramphenicol prophylaxis elicited immunity comparable to that of natural infection [21]. However, mass production of purified $O$. tsutsugamushi and retaining its stability upon storage are extremely difficult [22-24]. Therefore, whole cell vaccine products are unlikely to be economically feasible or suitable for manufacturing with current Good Manufacturing Practices Act standards of purity, potency, and lot-to-lot consistency. It is therefore, essential to develop a vaccine composed of genetically engineered materials which are easy to produce and capable of inducing protective immunity in human subjects after testing in relevant mouse models.

The $56 \mathrm{kDa}$ protein is the most immunodominant antigen of $O$. tsutsugamushi which accounts for $10-15 \%$ of the total cell protein [25-27]. This protein has four variable domains as well as five conserved domains and is responsible for the antigenic divergence of Orientia [7]. Almost every clinically diagnosed patient serum reacts with $56 \mathrm{kDa}$ antigen. Recombinant $56 \mathrm{kDa}$ protein and DNA plasmid carrying this protein gene have been shown to be protective in mice against homologous challenge by intraperitoneal injection (IP) or in 
cynomolgus monkey by intradermal injection [25-29]. The observed protective immune responses include antibody production, $\mathrm{T}$ cell proliferation, and production of IL-2 and IFN- $\gamma$ [28-32].

Since the scrub typhus disease is transmitted via the bite of an infected chigger and rodent is the natural reservoir of chigger, the best way to evaluate a vaccine candidate is using the chigger-mouse feeding model, which has been established at The Armed Forces Research Institute of Medical Sciences, Thailand (AFRIMS) [33-36]. A total of 12 Orientia infected and 3 uninfected chigger lines derived from three Leptotrombidium species are maintained [21,33,34] at AFRIMS $[33,34]$. Among these Orientia infected chigger lines; Lc-1 exhibited a $90 \%$ or higher infection rate [34]. This chigger line was identified as the most suitable line mimicking the natural challenge to evaluate vaccine candidates, i.e. infecting the mice by feeding a chigger in the mouse ear [34]. It was established previously that protein antigen based on the $56 \mathrm{kDa}$ protein could provide very good protection in mouse challenge by injection of live Orientia tsutsugamushi intraperitoneally $[31,32]$. However, the protective effect of the recombinant protein antigen in mouse directly infected by the bite of chigger, the natural vector of $O$. tsutsugamushi has not been evaluated. In order to evaluate whether recombinant protein antigen-based vaccine could provide protection in mouse challenged by an $O$. tsutsugamushi-infected chigger, we have produced the recombinant protein for $56 \mathrm{kDa}$ antigen based on the sequence of the Orientia strain carried by the chigger line Lc-1 (r56Lc-1).

This study described the production of r56Lc-1, the immunization regimen of out bred mice, and the observations of immunized mice after challenge in a chigger-mouse feeding model. The results have suggested that the protective efficacy ranged from $20-30 \%$, in addition to the effect of prolonged survival days in non survivors. This is the first report for the evaluation of a vaccine candidate in a natural challenge mouse model.

\section{Materials and Methods}

\section{Cloning and expression of $56 \mathrm{kDa}$ protein genes from $\mathrm{Lc}-1$}

Primers with built-in BamH1 and NdeI restriction sites for the 56 $\mathrm{kDa}$ protein gene from amino acid 80-456 (according to the sequence of Karp strain) of the open reading frame were designed based on the available DNA sequences of Karp strain in the NCBI database. Genomic DNA extracted from the liver of mouse, which was infected by Lc- 1 chiggers, was extracted as previously described using QiAamp DNA mini kit [35] and used in a polymerase chain reaction (PCR). The amplicons were cloned into a pET24a vector (Novagen, Wisconsin, USA). Colonies were selected and sequences of the amplicons were verified.

The plasmid was transformed into BL21 (DE3) cells (Invitrogen, Carlsbad, CA) for protein expression. BL21 (DE3) transformants containing correct amplicons were selected, grown in LB in the presence of $50 \mu \mathrm{g} / \mathrm{mL}$ kanamycin (Invitrogen) in a $37^{\circ} \mathrm{C}$ shaker and agitated at $200 \mathrm{rpm}$. The cells were induced with $1 \mathrm{mM}$ Isopropyl $\beta$ D-1-thiogalactopyranoside (IPTG, Sigma, St. Louis, MO) when OD600 reached 0.8-1.0. After induction for 19 hours, the cell culture was centrifuged at 4,000 rpm for 30 minutes in a SS34 Sorvall rotor to separate cells from LB medium. The cell pellet was stored in $-20^{\circ} \mathrm{C}$ until use.

\section{Extraction and solubilization of inclusion bodies containing the recombinant $56 \mathrm{kDa}$ (r56Lc-1) proteins}

The inclusion bodies were extracted as described previously [36]. Briefly, the cell pellet was thoroughly resuspended in $2 \%$ Deoxycholate (DOC, Sigma) in $20 \mathrm{mM}$ Tris-HCl, pH 7.5 (buffer A) and sonicated (Sonicator Ultrsonic Liquid Processor Model XL2020 with a standard tapered microtip) on ice. Following centrifugation at 8,000xg for 30 minutes at $4^{\circ} \mathrm{C}$, the resulting pellet was resuspended with $2 \mathrm{M}$ urea (Arcos Organics USA, Morris Plains, NJ) in buffer A, incubated with gentle rocking for $30 \mathrm{~min}$ and centrifuged again as described. The entire process was repeated with $4 \mathrm{M}, 6 \mathrm{M}$ and $8 \mathrm{M}$ urea in buffer $\mathrm{A}$. The extraction supernatants were analyzed by SDS-PAGE. The supernatant from $4 \mathrm{M}$ urea wash (approximately $10 \mathrm{ml}$ ) contained the majority of r56Lc- 1 proteins.

\section{Chromatographic purification of recombinant r56Lc- 1 in the presence of $6 \mathrm{M}$ urea}

The $4 \mathrm{M}$ urea supernatant containing r56Lc-1 protein was purified by DEAE anion-exchange chromatography with a 50 minute linear gradient of $0.30-0.70 \mathrm{M} \mathrm{NaCl}$ (Sigma-Aldrich, Missouri, USA) in the presence of $6 \mathrm{M}$ urea in buffer A (Buffer B). The purity of protein in each fraction was accessed by SDS-PAGE and fractions $>95 \%$ pure were pooled together. If the purity of protein was not satisfactory, a second run of DEAE chromatography was carried out after dialysis of the pooled fractions to remove $\mathrm{NaCl}$. The procedure for the second DEAE was similar to the first DEAE purification but the linear gradient was done in $0.30-0.60 \mathrm{M} \mathrm{NaCl}$ for 50 minutes. The final fractions containing r56Lc- 1 were evaluated for protein purity by SDSPAGE.

\section{Refolding of purified recombinant $56 \mathrm{kDa}$ proteins by dialysis}

The purified r56Lc-1 in $6 \mathrm{M}$ urea was refolded by sequential dialysis in decreasing concentrations of urea as described by Chao et al. [36]. The purified polypeptides at approximately $0.5 \mathrm{mg} / \mathrm{mL}$ in Buffer B were transferred into a dialysis bag $(24 \mathrm{~mm}$, molecular weight cutoff at 12,000 Daltons) and dialyzed sequentially against $4 \mathrm{M}, 2 \mathrm{M}$, and $0 \mathrm{M}$ urea in Buffer A for 30 min twice at each concentration of urea with gentle stirring. All dialysis procedures except the last step without urea were done at room temperature. The dialysis was continued overnight without urea in large excess of Buffer $\mathrm{A}$ at $4^{\circ} \mathrm{C}$ to remove traces of urea.

\section{Chigger line, animals and immunization}

Chiggers from the Leptotrombidium chiangraiensis line 1 (Lc-1) were selected for these challenge experiments because this line of chiggers exhibit stable infectivity of $90-100 \%$ in several generations [34]. Mus musculus, ICR strain female mice ordered from a Charles River Technology colony (BioLASCO, Taiwan) were maintained by the Department of Veterinary Medicine, AFRIMS, and used for this study as previously described in Lurchachaiwong et al. [34]. Each experimental group consisted of 40 mice each in 3 independent experiments, bringing the total number to 120 mice. Each experiment used 40 mice and the experiment was performed 3 times at 3 separate times. All animal procedures were approved by the AFRIMS Institutional Animal Care and Use Committee (IACUC). The refolded r56Lc-1 protein was dialyzed in $0.2 \mathrm{X}$ PBS and mixed with CpG 1826. Subsequently, equal volume of the protein-CpG solution and 
Citation: Ching WM, Lurchachaiwong W, Zhang Z, Awoyomi T, Chao CC, et al. (2014) Evaluation of a Recombinant Vaccine Candidate r56Lc-1 in a Chigger Challenge Mouse Model. J Vaccines Vaccin 5: 257. doi:10.4172/2157-7560.1000257

Page 3 of 8

Montanide were emulsified and $200 \mu \mathrm{L}$ of emulsified solution (containing $25 \mu \mathrm{g}$ of $\mathrm{r} 56 \mathrm{Lc}-1$ and $10 \mu \mathrm{g} \mathrm{CpG}$ ) was administered subcutaneously at 2 different sites $(100 \mu \mathrm{L} /$ site $)$ on the back for each immunization. For the groups receiving only the adjuvant, the same preparation was used except r56Lc-1 was replaced by $0.2 \mathrm{X}$ PBS. Mice were randomly selected from the colony for each experimental design group. Each mouse was immunized 3 times at 4 week intervals before challenge via chigger feeding.

\section{Chigger challenge}

Immunized mice were challenged by chigger bite 4 weeks after the last immunization. As previously described by Lurchachaiwong et al. [34], an individual chigger were placed into the inner ear of anesthetized females. Mice were placed in special holding cages during chigger feeding to restrict their movement and prevent chigger removal via scratching or grooming. A pan of water was placed under each cage to catch any chiggers fall from mice. The ear notching method was used to permanently identify each mouse. In general, the chiggers were fully engorged by day 2-3 and dropped off from the mouse (Figure 1). Forceps were used to remove chiggers still attached at day 3. Control mice were fed on by uninfected chiggers of $L$. chiangraiensis species. Dates of survival and symptoms onsets, such as the appearance of ruffled hair and the mobility of the mice, were recorded for 30 days after the challenge. Mice with limited mobility after moderate stimulation or those unable to move and had moderately or very rough hair were euthanized [37].

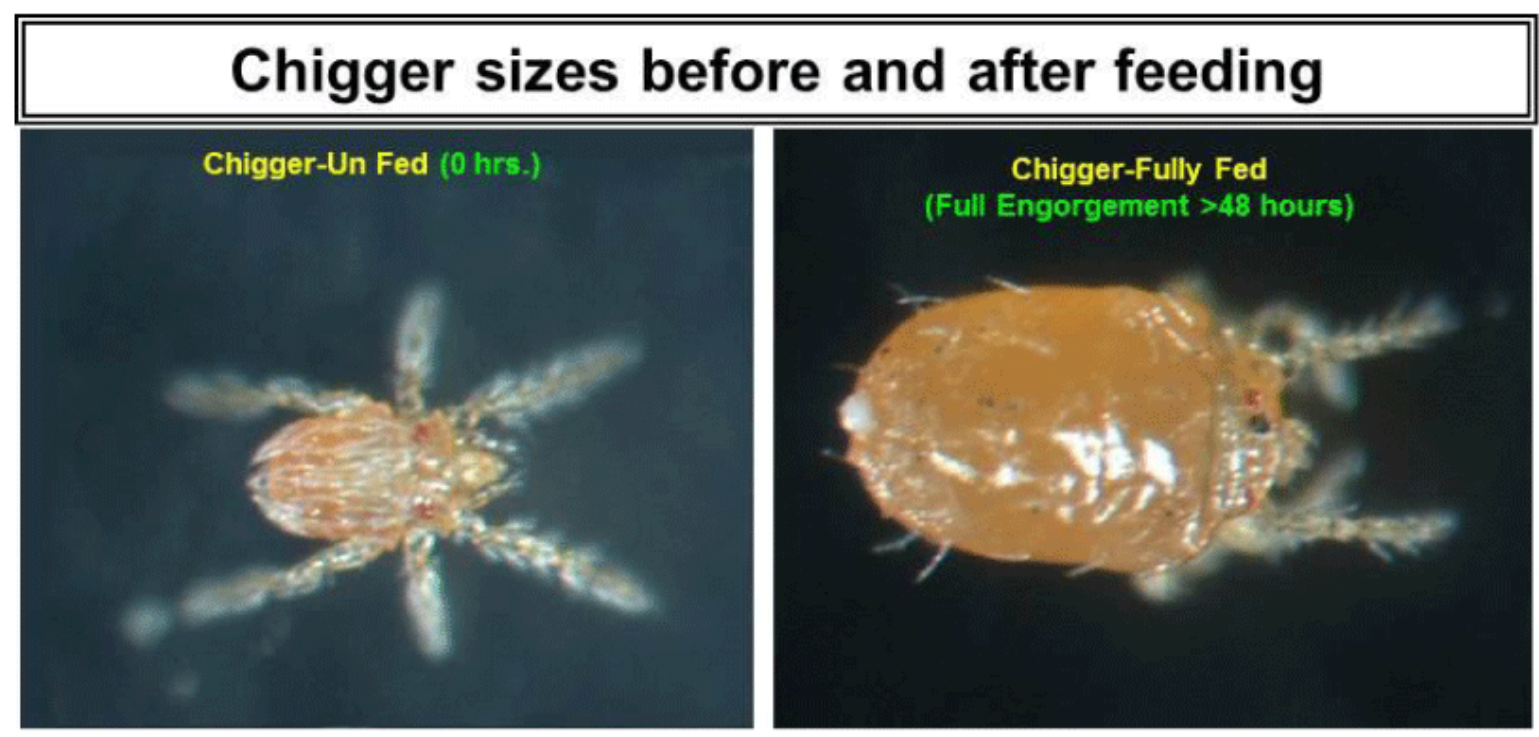

Figure 1: Chigger size and feeding status. Feeding status of chigger mite are shown over time, comparing an unfed mite (0 hour.) to a fully fed mite ( $>48$ hours). Pictures were taken at 60X magnification by Dr. Kriangkrai Lerdthusnee, Dept. of Entomology, AFRIMS.

\section{Antibody titer determination by ELISA}

The ELISA experiments were performed as previously described [36]. The concentration of r56Lc-1 protein antigen was adjusted to 0.3 $\mu \mathrm{g} / 100 \mu \mathrm{L}$ by $0.2 \mathrm{X}$ PBS based on previous experiments. The plate was coated at $4^{\circ} \mathrm{C}$ overnight. On the day of experiment, plates were first washed 3 times with $1 \mathrm{X}$ PBS containing $0.1 \%$ Tween 20 (1X PBST). The plates were then blocked with $200 \mu \mathrm{L} /$ well of $10 \%$ milk in $1 \mathrm{X}$ PBST and the plates were then incubated for 1 hour at room temperature. Mouse sera were diluted in 5\% milk in 1X PBST. For titration purposes, primary antibody was prepared for a serial dilution differed by a factor of 4 . Plates were incubated for 1 hour at room temperature. After incubation, plates were washed 3 times with $1 \mathrm{X}$ PBST. HRP conjugated goat anti-mouse IgG $(1: 5,000)$ or IgM $(1: 2,000)$ (Santa Cruz) was used as the secondary antibody. The secondary antibody was diluted in 5\% milk in $1 \mathrm{X}$ PBST and $100 \mu \mathrm{L} /$ well of diluted secondary antibody was applied and plates were incubated for 1 hour at room temperature. At the end of incubation, plates were washed 3 times with 1X PBST. A 1:1 ratio of ABTS $^{\oplus}$ Peroxidase Substrate Solution A and Peroxidase Substrate Solution B (Kirkegaard \& Perry Laboratories, Gaithersburg, MD) was prepared and added at
$100 \mu \mathrm{L} /$ well. Plates were incubated for 15-30 minutes in a dark drawer. Plates were read after incubation for 30 minutes at $405 \mathrm{~nm}-650 \mathrm{~nm}$ on a UVmax kinetic microplate reader (Molecular Devices, Sunnyvale, CA).

\section{Data analysis}

Days for onset of symptoms, the survival dates after onset of symptoms, and overall survival rates for control group and vaccinated group were calculated as the means of 3 independent experiments comprising of 40 mice per group using GraphPad Prism version 5.03 for Windows, GraphPad Software, San Diego California USA, www.graphpad.com. Error bars represent standard error or the mean $(+\mathrm{SEM})$

\section{Results}

\section{r56Lc-1 vaccine formulation}

In order to evaluate whether recombinant protein antigen-based vaccine could provide protection in mouse challenged by an $O$. 
tsutsugamushi-infected chigger, we prepared a recombinant protein antigen r56Lc-1. The gene was amplified from the Orientia strain infected chigger line 1 (and was named Orientia strain Lc-1). Our goal was to establish a homologous challenge model to exclude the consideration of factors due to heterogeneity in the $56 \mathrm{kDa}$ antigen. The r56Lc-1 protein, similar to previously expressed and purified 56 $\mathrm{kDa}$ proteins, was mainly present in inclusion bodies [38]. A similar purification procedure was applied and shown to be effective in generating a highly pure fraction of r56Lc-1 (Figure 2A). Once purified, the r56Lc-1 protein was evaluated for its reactivity with serum samples collected from mice infected by Lc-1 chigger or naïve chigger as shown on the western blot (Figure 2B). The purified recombinant protein was also evaluated via ELISA for detection of antibody responses elicited in mice challenged with live Lc-1 chiggers. Results confirmed that the protein was in a conformation recognized by the naturally-produced anti-56Lc- 1 antibody (data not shown). Furthermore, purifying with the HPLC DEAE column effectively eliminates bacterial LPS which may lead to unrelated immune activation that can interfere with the results. We also observed earlier that recombinant $56 \mathrm{kDa}$ proteins derived from Karp, Kato and Gilliam strains of $O$. tsutsugamushi precipitated in 1X PBS (Ching et al., unpublished results). Therefore, all the vaccine formulation was prepared by dialyzing the purified r56Lc- 1 in $0.2 \times$ PBS before making the emulsion with Montanide.
A

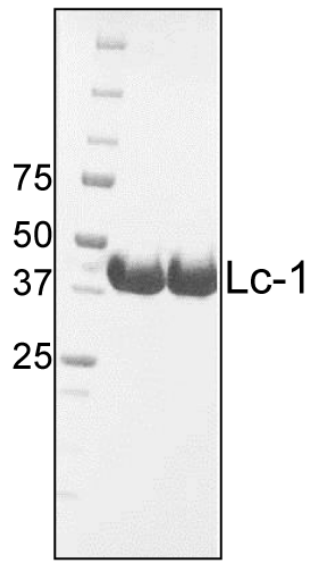

\section{B}

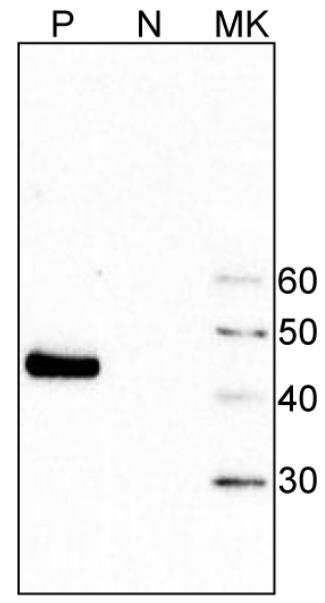

Figure 2: The r56Lc-1 protein after purification. (A) Two different pools of purified Lc-1 at $10 \mu \mathrm{g}$ per lane were loaded on to $4-20 \%$ gradient SDS-PAGE (Bio-Rad). After gel electrophoresis, the gel was stained with Gelcode Blue (Pierce) per manufacturer's instruction. The location of Lc-1 is shown as indicated between 37 $\mathrm{kDa}$ and $50 \mathrm{kDa}$ MW markers (B) Purified r56Lc-1 was evaluated to determine reactivity with serum samples collected from mice infected by Lc-1 chigger $(\mathrm{P})$ or naïve chigger $(\mathrm{N})$ as shown on the western blot. MK: molecular weight marker $(\mathrm{kDa})$.

\section{Evaluation of r56Lc-1 in chigger challenge model}

ICR mice were immunized with newly prepared emulsion (Montanide ISA-51) which contained $25 \mu \mathrm{g}$ of r56Lc- 1 and $10 \mu \mathrm{g}$ of CpG subcutaneously. The mice were immunized 3 times at 4 week intervals. The immunized mice were then challenged 4 weeks after the last immunization by an individual chigger from Lc-1 line of mite placed in the inner ear. After the mice were fed on by infected chiggers, all mice were observed daily and a record of their mobility and the roughness of their hair were taken until the end of study (day 30 after initiating chigger challenge). Delay of death and survival rate were also recorded and analyzed. Montanide emulsified in CpG 1826 was used as the adjuvant in the concentrations described above. Table 1 shows results for 3 independent challenge experiments. In each experiment, Group A comprised of ICR mice immunized with the adjuvant alone, Group B mice were immunized with the vaccine candidate. Within each group, there were 2 sub-groups: one group was challenged with uninfected chiggers (as controls) and the other challenged with infected chiggers. The survival rate for each group at the end of 30 days is shown in Table 1 . In each group, challenge by infected chiggers served as the mimic of the natural infection route. Experiments 1 and 2 presented $30 \%$ survival rates after immunization with the r56Lc- 1 vaccine candidate plus Montanide, while only $20 \%$ survived after the challenge in experiment 3.

\begin{tabular}{|c|c|c|c|c|}
\hline & & Immunogen & Chigger Challenge & $\begin{array}{l}\text { Survival } \\
\text { rate }\end{array}$ \\
\hline \multirow{4}{*}{ Expt. 1} & \multirow{2}{*}{ Group A } & Montanide & Uninfected chigger & $10 / 10$ \\
\hline & & Montanide & Lc-1 chigger & $0 / 10$ \\
\hline & \multirow{2}{*}{ Group B } & r56Lc-1+Montanide & Uninfected chigger & $10 / 10$ \\
\hline & & r56Lc-1+Montanide & Lc-1 chigger & $3 / 10$ \\
\hline \multirow{4}{*}{ Expt. 2} & \multirow{2}{*}{ Group A } & Montanide & Uninfected chigger & $10 / 10$ \\
\hline & & Montanide & Lc-1 chigger & $0 / 10$ \\
\hline & \multirow{2}{*}{ Group B } & r56Lc-1+Montanide & Uninfected chigger & $10 / 10$ \\
\hline & & r56Lc-1+Montanide & Lc-1 chigger & $3 / 10$ \\
\hline \multirow{4}{*}{ Expt. 3} & \multirow{2}{*}{ Group A } & Montanide & Uninfected chigger & $10 / 10$ \\
\hline & & Montanide & Lc-1 chigger & $0 / 10$ \\
\hline & \multirow{2}{*}{ Group B } & r56Lc-1+Montanide & Uninfected chigger & $10 / 10$ \\
\hline & & r56Lc-1+Montanide & LC-1 chigger & $2 / 10$ \\
\hline
\end{tabular}

Table 1: Evaluation of r56Lc-1 in chigger challenge model. ICR mice were immunized with either $25 \mu \mathrm{g}$ r56Lc-1 emulsion (Montanide) in the presence of $\mathrm{CpG}$ or adjuvant only before being fed on by either control chiggers or Orientia infected Lc-1 chiggers. Survival rates from 3 independent experiments are shown.

Representative data for the survival proportion analysis for experiment 2 ( 40 mice total) is shown in Figure 3A. No death was observed in the groups of mice challenged by uninfected chiggers, with or without the r56Lc-1 vaccine candidate. None of the mice immunized with adjuvants only survived the challenge by infected chiggers. Although the rate of survival is low, mice immunized with the vaccine fared better than those immunized with the adjuvant only. We measured the IgM and IgG responses of mice after three immunizations. The IgM titer was 1:6400 and IgG titer was greater than 1: 12800 . In previous studies, we monitored the antibody responses after each immunization of $\mathrm{r} 56 \mathrm{Kp}$, which has a $96 \%$ protein homology to r56Lc-1. The IgM titers were 1:1600 after one or two immunization and increased to 1:3200 after the third immunization. 
The IgG titers were all greater than 1:12800 regardless of the number of immunizations (Lerdthusnee et al., unpublished data). Since r $56 \mathrm{Kp}$ and r56Lc-1 exhibit high protein sequence homology, we expect that similar antibody responses would be observed after one or two immunizations with r56Lc-1 as we observed for r56Kp immunization.

(A)

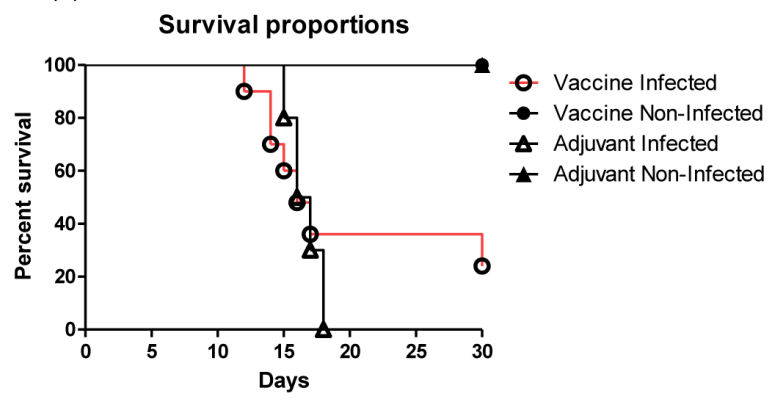

(B)

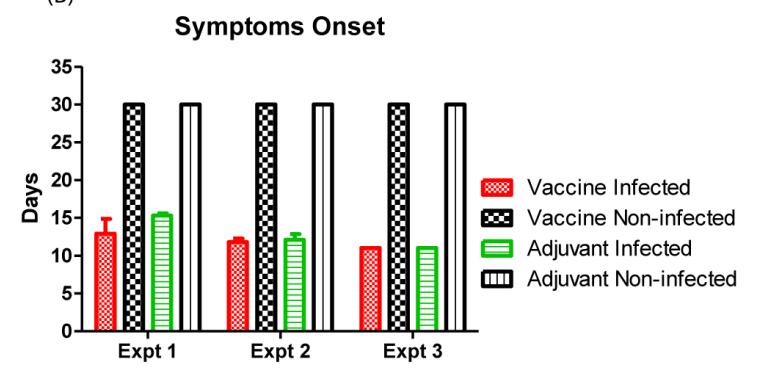

Figure 3: Survival rates and symptoms onset for immunized mice challenged by Lc-1 chiggers. ICR mice were immunized with either $25 \mu \mathrm{g}$ of newly engineered r56Lc-1 emulsion (Montanide) in the presence of $\mathrm{CpG}$ or adjuvant only subcutaneously 3 times at 4 week intervals, rested for 4 weeks before being fed on by either control chiggers or Orientia infected Lc-1 chiggers. Vaccine infected=immunized then infected with Lc-1 chiggers; Vaccine Non-Infected=immunized controls; Adjuvant Infected=immunized with adjuvant then infected with Lc-1 chiggers and Adjuvant NonInfected=immunized controls. (A) Survival rates are shown as representative data from 3 individual experiments (B) Symptoms onset are shown as means from 3 independent experiments of 40 mice per group. Error bars represent standard errors of the mean (SEM).

Symptoms onsets, as characterized by the roughness of their hair and degree of mobility, were recorded for mice challenged with either uninfected or infected chiggers for 30 days, after which the surviving mice were euthanized. As shown in Figure 3B, no symptoms were observed in all non-infected sub-groups, while the vaccinated mice challenged with infected chiggers began to exhibit symptoms at around day 11. All mice fed on by uninfected chiggers did not show any symptoms and no death occurred until the end of study. While those fed on by an infected chigger after immunization with the vaccine candidate, death occurred as early as day 8 . Thus, the data presented here demonstrates that r56Lc-1 provides $20-30 \%$ of homologous protection consistently upon challenge by an infected chigger. To further understand the intricacies of the protection provided by the vaccine candidate, we analysed the time between the initial observation of symptoms and death in all groups of mice. Since the non-infected groups showed no symptoms and all survived, the time between symptoms onset and death, are analysed for those mice challenged by infected chiggers. As shown in Figure 4, we can clearly see that the vaccine infected group survived 2-3 days longer than their adjuvant infected counterparts in experiments 2 and 3. The r56Lc-1 vaccinated mice were alive for an average of 7-8 days after the chigger challenge while those vaccinated with the adjuvant were alive for an average of 4-6 days. This r56Lc- 1 vaccine candidate was able to keep the mice alive longer than the adjuvant groups, a positive result that signifies that further studies are warranted to decipher the efficacy of r56Lc- 1 as an effective vaccine candidate.

\section{Time between symptoms onset and death}

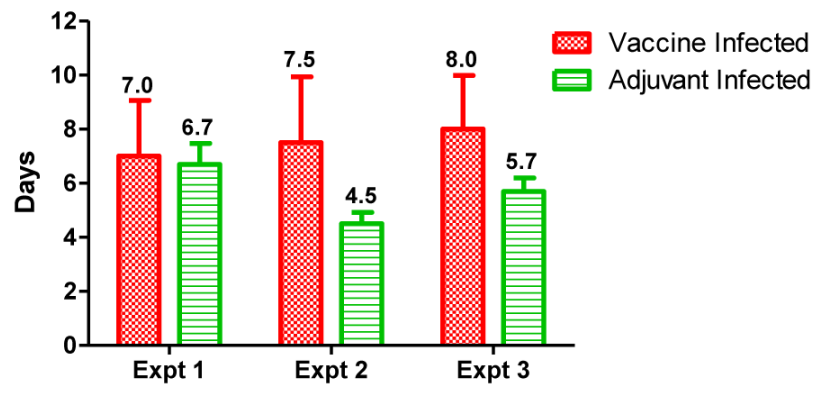

Figure 4: Time between symptoms onset and death. Mice immunized with either $25 \mu \mathrm{g}$ of r56Lc-1 emulsion+CpG or adjuvant only subcutaneously 3 times at 4 week intervals. The mice were rested for 4 weeks then fed on by either infected or control chiggers. The time between symptoms onset and death are shown as representative data from 3 individual experiments of 40 mice per group. Error bars represent standard errors of the mean (SEM). Vaccine infected=immunized then infected with Lc-1 chiggers; Adjuvant Infected=immunized with adjuvant then infected with Lc- 1 chiggers.

\section{Discussion}

We have produced and evaluated a promising vaccine candidate in a chigger challenge mouse model. This candidate, r56Lc- 1 is composed of the variable $56 \mathrm{kDa}$ outer membrane protein, the most immunodominant antigen of $O$. tsutsugamushi, which accounts for $10-15 \%$ of the total cell protein. Almost every clinically diagnosed patient serum reacts with $56 \mathrm{kDa}$ antigen [25,26,28,29]. $O$. tsutsugamushi exhibits considerable strain variation [13-16]. Homologous protection developed from natural infection persists for at least one year, but heterologous protection may remain for less than six months [21,39]. The $56 \mathrm{kDa}$ protein contains five conserved domains as well as four variable domains and is responsible for the antigenic divergence of Orientia. The $56 \mathrm{kDa}$ protein has been shown to be protective in mice against homologous challenge $[25,26,28,29]$. Both humoral and cell-mediated immune responses are important in protective immunity against scrub typhus [40-44]. Strong humoral and cellular immune responses were also induced in cynomolgus monkeys by $\mathrm{r} 56 \mathrm{Kp}$, with antigen-specific IgM and IgG produced to almost maximum levels within one week of a single immunization [31]. In addition, peripheral blood mononuclear cells from vaccinated animals showed an induction of antigen-specific proliferation and gamma interferon production [31]. All these observations have 
strongly suggested that $\mathrm{r} 56$ has the potential to be an effective vaccine candidate.

To investigate the protective capabilities of r56Lc-1 against Orientia infection in a chigger challenge model, we produced the vaccine and combined it with $\mathrm{CpG}$ and Montanide as the adjuvants. Montanide has been shown to elicit required cellular and humoral immune responses when used alone or in emulsions with other components [45-47]. It is also safe and well-tolerated in humans [47,48]. CpG has been shown to induce strong Th1 responses and to enhance vaccineinduced humoral immune responses [49]. CpG is also safe when used as an adjuvant in human vaccines [50,51]. ICR mice, like CD-1s, are outbred mice [52] and our choice of the ICR mouse strain was based on the premise that as outbred mice, the efficacy of this vaccine will provide valuable data that may have more similarities with what is expected in humans. Also, the lethal nature of the $O$. tsutsugamushi Lc-1 strain in ICR mice meant that we could better characterize the responses observed. Some of the effects of ICR infection after chigger feeding include reduction in food intake, lowered body temperatures, reduced mobility and loss of weight [34]. We observed reduced mobility and roughness in the fur in mice fed on by infected chiggers, irrespective of whether they were immunized by the adjuvant only or by the emulsified vaccine candidate.

Previously, we observed that $\mathrm{r} 56$ proteins were able to provide very good homologous protection but relatively poor heterologous protection in needle challenge mouse model. We anticipated a better survival rate by using the homologous immunogen in this chigger challenge model. However, our results indicated that although $3 / 10$ (30\%) of mice immunized with the vaccine survived the chigger challenge in experiments 1 and 2, only 20\% survived in the third experiment. This observation is in contrast to our previous experiments, where mice were immunized 3 times with $r 56 \mathrm{Kp}$ in a similar regimen as described in this study and $70-100 \%$ of the mice survived an intraperitoneal needle challenge (Ching et al. unpublished data). There are many different factors, such as the site of inoculation, the duration of infection upon chigger feeding, the components in chigger saliva, and interactions between the chigger vector and the host could have contributed to the discrepancies we observed. Also, during a natural chigger infection, there is the possibility of various strains of Orientia inhabiting the feeding mite, passing on various strains to the host. However, this is not the case with Lc-1, as only 1 sequence (94.2\% similar to Karp) was found in the analysed chiggers [35]. Even though the intraperitoneal route has been used extensively and has facilitated the elucidation of the mechanism of action of diseases $[15,28,29,53]$, alongside in the testing of vaccine candidates $[20,54,55]$, it only takes a couple of seconds to introduce the infectious organisms into mice. The chigger bite on the other hand, goes far beyond the initial break in the skin. The various components of the chigger saliva may play a role in the transmission of Orientia to the host. As an example of this concept, the sand fly (Leishmania infantum), which regurgitates infectious parasites and saliva into host skin during the blood meal, possesses saliva components that have been found to impair the classical pathway of the complement system in dogs, guinea pigs and rats [56]. It is therefore reasonable to hypothesize that the chigger may have similar effects on the host as it feeds on the epithelium. Another example is the tick saliva protein complex, 1xscS-1E1. After injected into the host during the blood meal, it inhibits platelet aggregation and clotting by interfering with thrombin [57]. This allows the tick to feed longer on the host. Another key point is the composition of the chigger saliva may depend on the stage of feeding as can be speculated from the huge size difference of the chigger at difference stage. Such effects were demonstrated during a study in cattle ticks. A difference in the amount of transporter proteins was observed between the saliva of fully or partially engorged cattle ticks [58]. Partially engorged ticks produced more of these transport proteins thought to transport active pharmacological agents from the tick to the host [58]. Although chiggers take the body fluid, from its host, this may also be the case for chigger mites. Another key point may be the composition of the chigger saliva depending on the stage of feeding. Such effects were demonstrated during a study in cattle ticks. A difference in the amount of transporter proteins was observed between the saliva of fully or partially engorged cattle ticks [58]. Partially engorged ticks produced more of these transport proteins thought to transport active pharmacological agents from the tick to the host [58]. This may also be the case for chigger mites considering the changes in the size of chigger from unfed to fully fed (Figure 1). The salivary components and the long-time feeding, which fosters interactions between vector-pathogen-host, may explain why the protective efficacy in chigger challenge model is not as good as those in needle challenge model.

In conclusion, our data demonstrated that $\mathrm{r} 56 \mathrm{Lc}-1$ provided $20-30 \%$ of protection consistently upon homologous challenge by an infected chigger. Since Orientia is an intracellular pathogen, the clearance of this pathogen may heavily depend on T-cell immune responses rather than B-cell immune responses. Vaccine candidate and adjuvants in formats which can promote mainly T-cell immunity may be able to improve the protective efficacy of the vaccine.

\section{Acknowledgements}

This work was supported by Work Unit Number (WUN) 6000.RAD1.J.A0311. The experiments reported herein were conducted in compliance with the Animal Welfare Act and approved by the AFRIMS Institutional Animal Care and Use Committee (12-13 AFRIMS). The opinions and assertions contained herein are the private ones of the authors and are not to be construed as official or as reflecting the views of the Department of the Navy, the Naval service at large, the Department of Defense, or the U. S. Government. Authors Wei-Mei Ching, Chien-Chung Chao and Anthony Schuster are employees (or military service members) of the U. S. Government. This work was prepared as part of official duties. Title 17 U.S.C. $\$ 105$ provides that 'Copyright protection under this title is not available for any work of the United States Government.' Title 17 U.S.C. $\$ 101$ defines a U.S. Government work as a work prepared by an employee of the U.S. Government as part of that person's official duties. The authors would like to thank Dr. Kriangkrai Lerdthusnee for his critical review of this manuscript.

\section{References}

1. Tamura A, Ohashi N, Urakami H, Miyamura S (1995) Classification of Rickettsia tsutsugamushi in a new genus, Orientia gen. nov., as Orientia tsutsugamushi comb. nov. Int J Syst Bacteriol 45: 589-591.

2. Brown GW, Robinson DM, Huxsoll DL, Ng TS, Lim KJ (1976) Scrub typhus: a common cause of illness in indigenous populations. Trans $\mathrm{R}$ Soc Trop Med Hyg 70: 444-448.

3. Paris DH, Blacksell SD, Nawtaisong P, Jenjaroen K, Teeraratkul A, et al. (2011) Diagnostic accuracy of a loop-mediated isothermal PCR assay for detection of Orientia tsutsugamushi during acute Scrub Typhus infection. PLoS Negl Trop Dis 5: e1307.

4. Yasunaga H, Horiguchi H, Kuwabara K, Hashimoto H, Matsuda S (2011) Delay in tetracycline treatment increases the risk of complications in 
Tsutsugamushi disease: data from the Japanese Diagnosis Procedure Combination database. Intern Med 50: 37-42.

5. Kumar K, Saxena VK, Thomas TG, Lal S (2004) Outbreak investigation of scrub Typhus in Himachal Pradesh (India). J Commun Dis 36: 277-283.

6. Chrispal A, Boorugu H, Gopinath KG, Prakash JA, Chandy S, et al. (2010) Scrub typhus: an unrecognized threat in South India - clinical profile and predictors of mortality. Trop Doct 40: 129-133.

7. Kelly DJ, Fuerst PA, Ching WM, Richards AL (2009) Scrub typhus: the geographic distribution of phenotypic and genotypic variants of Orientia tsutsugamushi. Clin Infect Dis 48 Suppl 3: S203-230.

8. Ahmad S, Srivastava S, Verma SK, Puri P, Shirazi N (2010) Scrub typhus in Uttarakhand, India: a common rickettsial disease in an uncommon geographical region. Trop Doct 40: 188-190.

9. Zhang S, Song H, Liu Y, Li Q, Wang Y, et al. (2010) Scrub typhus in previously unrecognized areas of endemicity in China. J Clin Microbiol 48: 1241-1244.

10. Lee YS, Wang PH, Tseng SJ, Ko CF, Teng HJ (2006) Epidemiology of scrub typhus in eastern Taiwan, 2000-2004. Jpn J Infect Dis 59: 235-238.

11. Izzard L, Fuller A, Blacksell SD, Paris DH, Richards AL, et al. (2010) Isolation of a novel Orientia species (O. chuto sp. nov.) from a patient infected in Dubai. J Clin Microbiol 48: 4404-4409.

12. Balcells ME, Rabagliati R, García P, Poggi H, Oddó D, et al. (2011) Endemic scrub typhus-like illness, Chile. Emerg Infect Dis 17: 1659-1663.

13. BELL EJ, BENNETT BL, WHITMAN L (1946) Antigenic differences between strains of scrub typhus as demonstrated by cross-neutralization tests. Proc Soc Exp Biol Med 62: 134-137.

14. Rights FL, Smadel JE (1948) Studies on scrib typhus; tsutsugamushi disease; heterogenicity of strains of R. tsutsugamushi as demonstrated by cross-vaccination studies. J Exp Med 87: 339-351.

15. Bennett BL, Smadel JE, Gauld RL (1949) Studies on scrub typhus; heterogeneity of strains of R. tsutsugamushi as demonstrated by crossneutralization tests. J Immunol 62: 453-461.

16. Kang JS, Chang WH (1999) Antigenic relationship among the eight prototype and new serotype strains of Orientia tsutsugamushi revealed by monoclonal antibodies. Microbiol Immunol 43: 229-234.

17. Watt G, Chouriyagune C, Ruangweerayud R, Watcharapichat P, Phulsuksombati D, et al. (1996) Scrub typhus infections poorly responsive to antibiotics in northern Thailand. Lancet 348: 86-89.

18. Mathai E, Rolain JM, Verghese GM, Abraham OC, Mathai D, et al. (2003) Outbreak of scrub typhus in southern India during the cooler months. Ann N Y Acad Sci 990: 359-364.

19. Kekcheyeva N (1968) Preventive immunization against tsutsugamushi fever. J Hyg Epidemiol Microbiol Immunol 12: 14-17.

20. Eisenberg GH, Osterman JV (1977) Experimental scrub typhus immunogens: gamma-irradiated and formalinized rickettsiae. Infect Immun 15: 124-131.

21. Smadel JE, Ley Hl, Diercks FH, Paterson PY, Wisseman CI, et al. (1952) Immunization against scrub typhus: duration of immunity in volunteers following combined living vaccine and chemoprophylaxis. Am J Trop Med Hyg 1: 87-99.

22. Eisenberg GH, Osterman JV (1978) Effects of temperature on the stability of Rickettsia tsutsugamushi and gamma-irradiated scrub typhus immunogens. Infect Immun 22: 298-300.

23. Bovarnick MR, Miller JC, Snyder JC (1950) The influence of certain salts, amino acids, sugars, and proteins on the stability of rickettsiae. J Bacteriol 59: 509-522.

24. Bozeman FM, Hopps HE, Danauskas JX, Jackson EB, Smadel JE (1956) Study on the growth of Rickettsiae. I. A tissue culture system for quantitative estimations of Rickettsia tsutsugamushi. J Immunol 76: 475-488.

25. Ohashi N, Tamura A, Suto T (1988) Immunoblotting analysis of antirickettsial antibodies produced in patients of Tsutsugamushi disease. Microbiol Immunol 32: 1085-1092.
26. Tay ST, Rohani MY, Ho TM, Devi S (2002) Antigenic types of Orientia tsutsugamushi in Malaysia. Southeast Asian J Trop Med Public Health 33: 557-564.

27. Chen HW, Zhang Z, Huber E, Chao CC, Wang H, et al. (2009) Identification of cross-reactive epitopes on the conserved 47 -kilodalton antigen of Orientia tsutsugamushi and human serine protease. Infect Immun 77: 2311-2319.

28. Seong SY, Huh MS, Jang WJ, Park SG, Kim JG, et al. (1997) Induction of homologous immune response to Rickettsia tsutsugamushi Boryong with a partial 56-kilodalton recombinant antigen fused with the maltosebinding protein MBP-Bor56. Infect Immun 65: 1541-1545.

29. Yu Y, Wen B, Wen B, Niu D, Chen M, et al. (2005) Induction of protective immunity against scrub typhus with a 56-kilodalton recombinant antigen fused with a 47-kilodalton antigen of Orientia tsutsugamushi Karp. Am J Trop Med Hyg 72: 458-464.

30. Seong SY, Kim HR, Huh MS, Park SG, Kang JS, et al. (1997) Induction of neutralizing antibody in mice by immunization with recombinant $56 \mathrm{kDa}$ protein of Orientia tsutsugamushi. Vaccine 15: 1741-1747.

31. Chattopadhyay S, Jiang J, Chan TC, Manetz TS, Chao CC, et al. (2005) Scrub typhus vaccine candidate Kp r56 induces humoral and cellular immune responses in cynomolgus monkeys. Infect Immun 73: 5039-5047.

32. Ni YS, Chan TC, Chao CC, Richards AL, Dasch GA, et al. (2005) Protection against scrub typhus by a plasmid vaccine encoding the 56KD outer membrane protein antigen gene. Am J Trop Med Hyg 73: 936-941.

33. Lerdthusnee K, Khlaimanee N, Monkanna T, Sangjun N, Mungviriya S, et al. (2002) Efficiency of Leptotrombidium chiggers (Acari: Trombiculidae) at transmitting Orientia tsutsugamushi to laboratory mice. J Med Entomol 39: 521-525.

34. Lurchachaiwong W, Monkanna T, Leepitakrat S, Ponlawat A, Sattabongkot J, et al. (2012) Variable clinical responses of a scrub typhus outbred mouse model to feeding by Orientia tsutsugamushi infected mites. Exp Appl Acarol 58: 23-34.

35. Takhampunya R, Tippayachai B, Promsathaporn S, Leepitakrat S, Monkanna T, et al. (2014) Characterization based on the 56-Kda typespecific antigen gene of Orientia tsutsugamushi genotypes isolated from Leptotrombidium mites and the rodent host post-infection. Am J Trop Med Hyg 90: 139-146.

36. Chao CC, Huber ES, Porter TB, Zhang Z, Ching WM (2011) Analysis of the cross-reactivity of various $56 \mathrm{kDa}$ recombinant protein antigens with serum samples collected after Orientia tsutsugamushi infection by ELISA. Am J Trop Med Hyg 84: 967-972.

37. Lurchachaiwong W, Chan TC, Richards AL, McCardle W, Schuster AL (2014) Establishment of Orientia tsutsugamushi Lc-1 (Rickettsiales: Rickettsiaceae) infection in ICR outbred mice (Rodentia: Muridae) by needle challenge. J Med Entomol 51: 658-660.

38. Yang Q, Ching WM, Jiang J, Lousteau L, Richards AL (2003) An improved method for the purification and refolding of $\mathrm{r} 56-\mathrm{kDa}$ proteins from Gilliam and Kato strains of Orientia tsutsugamushi. Ann N Y Acad Sci 990: 375-385.

39. Smadel JE, Ley HL, Diercks FH, Traub R (1950) Immunity in scrub typhus: resistance to induced reinfection. AMA Arch Pathol 50: 847-861.

40. Jerrells TR, Osterman JV (1982) Host defenses in experimental scrub typhus: delayed-type hypersensitivity responses of inbred mice. Infect Immun 35: 117-123.

41. Jerrells TR, Osterman JV (1983) Development of specific and crossreactive lymphocyte proliferative responses during chronic immunizing infections with Rickettsia tsutsugamushi. Infect Immun 40: 147-156.

42. Hickman CJ, Stover CK, Joseph SW, Oaks EV (1993) Murine T-cell response to native and recombinant protein antigens of Rickettsia tsutsugamushi. Infect Immun 61: 1674-1681.

43. Seong SY, Kim MK, Lee SM, Odgerel Z, Choi MS, et al. (2000) Neutralization epitopes on the antigenic domain II of the Orientia tsutsugamushi $56-\mathrm{kDa}$ protein revealed by monoclonal antibodies. Vaccine 19: 2-9. 
Citation: Ching WM, Lurchachaiwong W, Zhang Z, Awoyomi T, Chao CC, et al. (2014) Evaluation of a Recombinant Vaccine Candidate r56Lc-1 in a Chigger Challenge Mouse Model. J Vaccines Vaccin 5: 257. doi:10.4172/2157-7560.1000257

Page 8 of 8

44. Hanson BA (1983) Effect of immune serum on infectivity of Rickettsia tsutsugamushi. Infect Immun 42: 341-349.

45. Yamshchikov GV, Barnd DL, Eastham S, Galavotti H, Patterson JW, et al. (2001) Evaluation of peptide vaccine immunogenicity in draining lymph nodes and peripheral blood of melanoma patients. Int J Cancer 92: 703-711.

46. Arevalo-Herrera M, Vera O, Castellanos A, Cespedes N, Soto L, et al (2011). Preclinical vaccine study of Plasmodium vivax circumsporozoite protein derived-synthetic polypeptides formulated in montanide ISA 720 and montanide ISA 51 adjuvants. Am J Trop Med Hyg 84: 21-27.

47. Kusi KA, Remarque EJ, Riasat V, Walraven V, Thomas AW, et al. (2011) Safety and immunogenicity of multi-antigen AMAl-based vaccines formulated with CoVaccine HTâ,\$ and Montanide ISA 51 in rhesus macaques. Malar J 10: 182.

48. Aucouturier J, Dupuis L, Deville S, Ascarateil S, Ganne V (2002) Montanide ISA 720 and 51: a new generation of water in oil emulsions as adjuvants for human vaccines. Expert Rev Vaccines 1: 111-118.

49. Bode C, Zhao G, Steinhagen F, Kinjo T, Klinman DM (2011) CpG DNA as a vaccine adjuvant. Expert Rev Vaccines 10: 499-511.

50. Sagara I, Ellis RD, Dicko A, Niambele MB, Kamate B, et al. (2009) A randomized and controlled Phase 1 study of the safety and immunogenicity of the AMA1-C1/Alhydrogel + CPG 7909 vaccine for Plasmodium falciparum malaria in semi-immune Malian adults. Vaccine 27: 7292-7298.

51. Sagara I, Dicko A, Ellis RD, Fay MP, Diawara SI, et al. (2009) A randomized controlled phase 2 trial of the blood stage AMA1-C1/ Alhydrogel malaria vaccine in children in Mali. Vaccine 27: 3090-3098.
52. Aldinger KA, Sokoloff G, Rosenberg DM, Palmer AA, Millen KJ (2009) Genetic variation and population substructure in outbred CD-1 mice: implications for genome-wide association studies. PLoS One 4: e4729.

53. Groves MG, Osterman JV (1978) Host defenses in experimental scrub typhus: genetics of natural resistance to infection. Infect Immun 19: 583-588.

54. Eisenberg GH Jr, Osterman JV (1979) Gamma-irradiated scrub typhus immunogens: broad-spectrum immunity with combinations of rickettsial strains. Infect Immun 26: 131-136.

55. Eisenberg GH Jr, Osterman JV (1978) Gamma-irradiated scrub typhus immunogens: development and duration of immunity. Infect Immun 22: 80-86.

56. Mendes-Sousa AF, Nascimento AA, Queiroz DC, Vale VF, Fujiwara RT, et al. (2013) Different host complement systems and their interactions with saliva from Lutzomyia longipalpis (Diptera, Psychodidae) and Leishmania infantum promastigotes. PLoS One 8: e79787.

57. Ibelli AM, Kim TK, Hill CC, Lewis LA, Bakshi M, et al. (2014) A blood meal-induced Ixodes scapularis tick saliva serpin inhibits trypsin and thrombin, and interferes with platelet aggregation and blood clotting. Int J Parasitol 44: 369-379.

58. Tirloni L, Reck J, Terra RM, Martins JR, Mulenga A, et al. (2014) Proteomic Analysis of Cattle Tick Rhipicephalus (Boophilus) microplus Saliva: A Comparison between Partially and Fully Engorged Females. PLoS One 9: e94831. 